\title{
CÁLCULO APROXIMADO DE COEFICIENTES DE PERMEABILIDADE EM ROCHAS SEDIMENTARES DE AQUIFEROS
}

\author{
MOACYR DE CARVALHO *
}

\section{RESUMO}

O presente trabalho completa um anterior já publicado na Rev. IG, v. 1, n. 1. jan./jun. 1980, versando sobre o cálculo aproximado de coeficientes de permeabilidade dos diversos estratos de sedimentos que, geralmente, compõem um aquífero.

Trata.se de um método que se baseia fundamentalmente na representação de um fenômeno mecânico ou seja da percolação da água em um meio poroso, através de um sistema de equações lineares.

Embora a parte já publicada e a presente estejam ligadas pela mesma idéia central, são dois procedimentos distintos no que concerne à geração do sistema de equações.

\section{ABSTRACT}

The present paper is a complement of a previous work published in the Rev. IG, v. 1, n. 1, Jan./Jun. 1980, dealing about approximate valuation of permeability coefficients about several sediments beddings in which generally aquifer are constituted.

The method is based fundamentally in the physical phenomenon presentation, that is, the water filtration in the porous environment, through a system of linear equations.

Although the information published and the present work are linked by the same central idea, there are two different proceedings in the equations system generation.

\section{INTRODUÇÃO}

Na Revista do Instituto Geológico, v. I, n. 1 1980, apresentamos uma metodologia com a finalidade de se calcular aproximadamente os coeficientes de permeabilidade de um aquífero, pela formulação de um sistema de equações lineares, mediante certas restrições baseadas nas seguintes suposições:

- a estrutura do aquífero tem certa uniformidade, isto é, ocorre segundo camadas contínuas e com um certo grau de uniformidade sedimentológica;

- cada camada de sedimentos comporta-se como aquífero livre e independente;

- as camadas isoladas do aquífero obedecem à equação de DUPUIT;

- o rebaixamento total do lençól, sob bombeamento, se distribue proporcionalmente à espessura dos sedimentos;

\footnotetext{
* Engenheiro - Pesquisador Científico - Assess oria de Programação - Instituto Geológico.
} 
- o diâmetro do poço tubular é constante e o raio de influência é tomado aproximadamente em função da fração textural principal dos sedimentos presentes em cada camada (areia, argila, silte, etc.).

Estas hipóteses de base, evidentemente necessárias para se proceder tal avaliação, poderão ser melhoradas. Por exemplo, o rebaixamento total distribuído proporcionalmente à espessura do aquífero poderá ainda considerar uma definida escala de valores que guarde uma relação com a composição e a distribuição granulométrica dos estratos.

Como a investigação da metodologia comporta ainda uma idéia fundamental, já que está interessada em obter valores aproximados de permeabilidade, é certo que estes valores são passíveis de serem melhorados em limites satisfatórios para a solução problema em foco, pois que se trata de um modelo sempre possível de ser modificado de acordo com os resultados de sua aplicação. Em hidrologia é frequente a sugestão de vários modelos para a solução de problemas reais, com menor ou maior grau de aproximação nos resultados.

O método proposto consistiu em supor que a vazão $Q$, obtida em teste de bombeamento de produção de um poço tubular, pode ser decomposta segundo a expressão:

$$
\Sigma \mathrm{Qi}=\mathrm{Q} \quad \mathrm{i}=1,2, \ldots, \mathrm{n} \quad \mathrm{o}
$$
que é sempre possível, onde o índice genérico $i$ designa uma camada arbitrária do aquífero. A partir de $n$ poços tubulares situados numa área restrita é possível obter-se o sistema de equações lineares

$$
\begin{gathered}
\Sigma a_{i j K j}-Q j=0 \quad(1) \text { ou } A K=Q \\
i, j=1,2, \ldots \ldots, n
\end{gathered}
$$

de solução única sob a condição de que a matriz quadrada $A$ tenha o seu determinante Det $(A) \neq 0$, onde os aij são os elementos da matriz dos coeficientes do sistema.
Para o cálculo dos coeficientes aij admitiu-se a fórmula

aij $=\frac{\pi{ }^{2}}{24 \mathrm{H}^{2}} \frac{(\mathrm{H}-\mathrm{h})^{2}}{(\ln R \mathrm{j}-\ln \mathrm{r})}(2)$ como consequência das hipóteses iniciais e da expressão (1) acima, onde:

lj é a espessura da camada de ordem $\mathrm{j}$;

$(\mathrm{H}-\mathrm{h})^{2}=$ ao quadrado do rebaixamento total;

$\mathrm{H}=$ altura do plano representado pelo nível estático a partir do plano que passa pelo fundo do poço;

$R_{j}$ e $r$, respectivamente, o raio de influência e o raio do poço, sendo o primeiro relacionado com a fração principal do sedimento da camada $j$ (predominância percentual de areia, argila ou silte...).

Note-se que (2) é parte da equação de Dupuit, ou seja, Qi = aij Kj, onde $\mathrm{Kj}$ é a incognita procurada, o que permite a formulação do sistema (1). Também os coeficientes estão ajustados para exprimir a vazão e o coeficiente de permiabilidade em unidades compatíveis.

\section{OBJETIVO}

Este trabalho visa mostrar a flexibilidade do método em questão, na demonstração de que o sistema de equações (1) poderá ser gerado no ensaio de teste de um único poço tubular se, a partir da identificação dos estratos no curso da perfuração, desde que se introduza variações da vazão de $Q$ no poço testado, tantas vezes quanto seja o número de estratos de sedimentos registrados.

Ora, a variação de $\mathrm{Q}$ mediante uma modificação na operação de bombeamento traz como consequência uma variação no rebaixamento, já que este é uma função de $Q$ em certos limites. 
Por exemplo, a potência de uma bomba centrífuga se exprime por:

$$
\text { No }=\frac{\gamma \mathrm{QHp}}{75 \mathrm{n}} \quad \text { (n rendimento }
$$

da bomba).

$\mathrm{H}_{\mathrm{P}}$ com base no teorema de Bernoulli e em simplificações se representa por $\frac{p_{2}-p_{1}}{\gamma}$ com variação segundo perdas de carga no sistema, neste caso, perdas de energia específica de pressão. Assim se pode por este procedimento modificar $Q$ e, por consequência, o rebaixamento, isto é, o valor de h (nível dinâmico) é uma variável permanecendo $\mathrm{H}$ constante.

\section{JUSTIFICATIVA}

A medida de coeficientes de permeabilidade, mesmo que aproximada dentro de limites razoáveis, é uma questão de interesse no conhecimento da estrutura de aquíferos bem como do movimento da água através das rochas, do qual depende o seu manejo racional, pois isto tem implicações na econômia regional, tanto maiores quanto mais intensa for a exploração da água procedente do aquífero. Pois, neste caso, é necessário manter em relativo equilíbrio a capacidade de recarga do aquífero e a extração de água através de poços tubulares.

\section{METODOLOGIA}

A presente investigação, tal como a concebemos e justificamos, segue uma metodologia geralmente adotada pelos pesquisadores que se ocupam desse segmento do ciclo hidrológico. Ela tem sido proposta, mais, por modelos matemáticos, o que é compreensível, em face das dificuldades impostas pelo método direto. Este, via de regra, exige fortes investimentos, grande dispêndio de tempo, além de sua limitação dada as dimensões físicas do próprio problema.
Assim a metodologia adotada foi a de associar um modelo matemático ao fenômeno físico considerado. que pudesse ter aplicação. Consideramos, por outro lado, que os ensaios de permeabilidade em laboratório, ainda que mais precisos, são obtidos de amostras de rochas e a sua quantidade a par de outras dificuldades não permitem in-

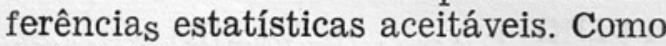
dificuldade pode-se mencionar a obtenção de amostras de material não consolidado.

\section{ANÁLISE DO PROBLEMA}

A nossa análise vai estar centrada na comprovação do fato de que se um sistema de equações lineares de $n$ equações e $n$ incognitas, gerado pela observação das variaçõe $e_{S}$ de vazão e do rebaixamento total de um único poço tubular, são independentes entre si e constitue um sistema consistente, isto é, duas equações quaisquer do sistema não são combinações lineares.

Adotemos a linguagem da álgebra matricial para brevidade da exposição. O sistema (1) será designado:

$$
\mathrm{AK}=\mathrm{Q},
$$

sendo A a matriz dos coeficiente $\mathrm{a}_{\mathrm{ij}}$ do sistema, associada ao det (A). Q e $K$ representam os vetores coluna respectivamente das vazões observadas e dos coeficiente de $_{S}$ permeabilidade a serem determinados pela solução do sistema (1).

Para a nossa finalidade é suficiente comprovar que no det (A) não ocorrem linhas oü colunas proporcionais, nem nulas. Pois neste caso det (A) $\neq 0$ e o sistema admite solução única e o modelo tem completa flexibilidade em sua aplicação.

Tomadas duas linhas ou duas coluna $_{S}$ arbitrárias do $\operatorname{det}(\mathrm{A})$, deverão ser verificadas as relações seguintes: 


$$
\frac{a_{i l}}{a_{i+1,1}} \neq \frac{a_{i}}{a_{i}+1,2} \neq \cdots \neq \frac{a_{i n} e}{a_{i+1, n}} \frac{a_{1 j}}{a_{1, j+1}} \neq \frac{a_{2 j}}{a_{2, j+1}} \neq \cdots \frac{a_{n j}}{a_{n, j+1}}
$$

Para situar escrevemos o sistema $A K=Q$ na forma expandida

$$
\begin{aligned}
& \mathrm{a} l \mathrm{KK} 1+\ldots \ldots+\mathrm{aljK}+\ldots \ldots+\operatorname{alnKn}=\mathrm{Q} 1 \\
& a_{i l K l}+\ldots \ldots+a_{i j K j}+\ldots \ldots+\operatorname{ainKn}=Q \cdots \cdots \cdots
\end{aligned}
$$

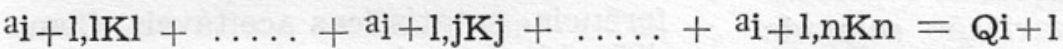

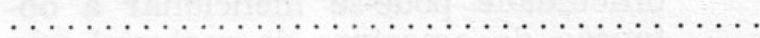

$$
\begin{aligned}
& a_{n l K I}+\ldots \ldots+a_{n j K j}+\ldots \ldots+a_{n n K n}=Q n
\end{aligned}
$$

observando que o índice $j$ indica a posição sucessiva das camadas de sedimento e também a coluna de ordem $j$ do determinante det (A), enquanto que o índice $i$ designa a ordem das equiações correspondentes à vazão genérica $Q_{i}$ e a linha $i$ do determinante.

Demonstremos que variando $Q$ por um incremento $\Delta Q$, ou seja $\mid \mathrm{Qi}+1$ - Qi $\mid=\Delta \mathrm{Q}$, em correspondência com a variação do rebaixamento do lençól, obteremos duas equações do sistema, cujos coeficientes não são proporcionais, isto é, não são combinações lineares entre si.

Tomemos arbitrariamente dois coeficientes $a_{i j}$ e $a_{i}+1, j$ (situados na mesma coluna $j$ do $\operatorname{det}(\mathrm{A})$ e mostremos que não se verifica a relação:

$$
\frac{a_{i j}}{a_{i+1, j}}=m \text { ou } a_{i j}=a i+1, j \times m \text {, }
$$

onde $m$ é uma constante, e por consequência sendo

$$
\mathrm{Qi}=\mathrm{a}_{\mathrm{ij}} \mathrm{Kj} \text { e } \mathrm{Qi}+1=\mathrm{a}_{\mathrm{i}}+1, \mathrm{j} \mathrm{Kj}
$$

tem-se Qi aij como

$$
\frac{\mathrm{Qi}+1}{\mathrm{Qi}}=\frac{\mathrm{ai}+1, \mathrm{j}}{\mathrm{a} i \mathrm{~m}}
$$

se quer demonstrar.

Nós temos admitido para cálculo dos coeficientes aij a expressão seguinte:

$a_{i j}=\mathrm{C} \times(\mathrm{H}-\mathrm{h})^{2}$ onde para a camada $j$ considerada

$$
\mathrm{C}=\frac{\pi^{\mathrm{l}^{2}} \quad \text { é uma constante, }}{24 \mathrm{H}^{2}(\ln \mathrm{Rj}-\ln r)}
$$

Tomando dois coeficientes arbitrários da coluna $j$ do det $(\mathrm{A})$, podemos escrever:

$$
\frac{a i j}{a_{i}+1, j}=\frac{(H-h i)^{2}}{(H-h i+1) 2} \neq m
$$

onde $h$ é uma variável relacionada à variação de $Q$.

A relação anterior mostra a inexistência de proporcionalidade entre $a_{i j}$ e $a_{i}+1, j$, pois o lado direto da expressão varia de acordo com uma curva durante uma variação contínua de Q. Sendo os coeficientes tomados arbitrariamente resulta que entre as linhas de $\operatorname{det}(\mathrm{A})$ não existem combinações lineares.

O mesmo acontece com as colunas do determinante considerado. Na verdade, tomando novamente a fórmula de cálculo de $a_{i j}$, verificamos que dois elementos quaisquer da linha $i$ diferem entre si ou pela espessura da camada de sedimento correspondente, ou então pela diferença $\ln R_{j}-\ln r$, visto que $R_{j}$ varia de camada para camada (granulometrias diferentes).

$$
\text { Logo } \frac{a_{i j}}{a_{i, j+1}} \neq m \text { onde } m
$$

indica uma constante.

A conclusão final é que o sistema gerado pela via indicada é consistente, pois implica em $\operatorname{det}(A) \neq 0$ e a metodologia exposta em nosso trabalho constante na Rev. IG, v. I, n. 1 1980 , se estende para o caso em que o número de poços tubulares se reduza a um, o que é o escopo deste trabalho. 


\section{CONSIDERAÇÕES FINAIS}

As investigações, que estamos realizando em torno de aspectos hidrogeológicos de aquíferos, relacionam-se com a avaliação de coeficientes de permeabilidade e contêm certo aspecto teórico, o que é uma característica geral no desenvolvimento de modelos visando representar um fenômeno complexo da natureza.

Outra faceta na abordagem destas questões são as hipóteses colocadas em seus fundamentos, cuja validade e posteriores ajustagem provêm dos testes experimentais a que são submetidas. Por outro lado existem problemas tais, que pela sua extrema complexidade, somente são viáveis de solução dentro de certos limites de precisão.

No caso examinado, o fenômeno de percolação da água em interstícios de rochas apresenta contínua variação, com nítido cunho probabilístico, em virtude de quantidade de variáveis em ação.

Porém nas avaliações que entram em jogo variáveis que interferem no ciclo hidrológico, pode-se chegar a aproximações de parâmetros com a precisão necessária para se propor o problema considerado, evidentemente sem se procurar senão a precisão possível.

O modelo que desenvolvemos está dentro dessas limitações e a sua aplicabilidade dependerá não somente da maior ou menor exatidão com que se obtenham os dados necessários para a geração do sistema, mas também da ajustagem mais real das hipóteses de base.

Um número suficiente de testes experimentais constitue a via necessária para se melhorar o método.

As medidas necessárias para a formação das equações do sistema (1) são: as vazões incrementadas, os correspondentes valores do nível dinâmico estabilizado e a granulometria do material interceptado pelo poço e suas espessuras. A granulometria dos sedimentos é necessária para a avaliação do raio de influência, como já foi explanado na primeira parte do trabalho publicado.

As simplificações, que o método comporta, consiste na possível redução do número de equações do sistema (1). Assim, se ocorrerem camadas de sedimentos alternados, onde os valores das frações prncipais dos sedimentos (predomínio percentual de silte, areia, argila, etc.) estejam próximos entre si, essas camadas poderão ser assimiladas a uma única com espessura total igual à soma das espessuras parciais. No caso de sedimentos argilosos, estes poderão ser excluídos devido à baixa permeabilidade da argila.

No entanto é necessário que os pares de valores Qi e hi, bem como as espessuras dos sedimentos sejam determinados com a melhor precisão possível para que os valores dos coeficientes de permeabilidade se aproximem melhor de seus valores reais. 